\title{
Pathophysiology of chronic bacterial osteomyelitis. Why do antibiotics fail so often?
}

\author{
J Ciampolini, K G Harding
}

\begin{abstract}
In this review the pathophysiology of chronic bacterial osteomyelitis is summarised, focusing on how bacteria succeed so often in overcoming both host defence mechanisms and antibiotic agents. Bacteria adhere to bone matrix and orthopaedic implants via receptors to fibronectin and to other structural proteins. They subsequently elude host defences and antibiotics by "hiding" intracellularly, by developing a slimy coat, or by acquiring a very slow metabolic rate. The presence of an orthopaedic implant also causes a local polymorphonuclear cell defect, with decreased ability to kill phagocytosed bacteria. Osteolysis is determined locally by the interaction of bacterial surface components with immune system cells and subsequent cytokine production. The increasing development of antibiotic resistance by Staphylococcus aureus and $S$ epidermidis will probably make conservative treatment even less successful than it is now. A close interaction between orthopaedic surgeons and physicians, with combined medical and operative treatment, is to be commended.

(Postgrad Med f 2000;76:479-483)
\end{abstract}

Keywords: osteomyelitis; joint replacement infection; antibiotics; osteolysis

Acute bacterial osteomyelitis carried a 50\% mortality in the preantibiotic era because of overwhelming sepsis with metastatic abscesses. ${ }^{1}$ Although antimicrobial drugs have dramatically changed the prognosis of the acute haematogenous form, chronic bacterial osteomyelitis remains a challenging medical problem. Despite advances in antibiotic development, it all too often remains refractory to chemotherapeutic treatment alone. ${ }^{2}$ The rapid development of antibiotic resistance by Staphylococcus spp will probably demand more frequently a strategy of combined medical and surgical treatment in the near future.

In this review we analyse the current understanding of chronic osteomyelitis by focusing on how bacteria succeed in overcoming both host defence mechanisms and the antibiotic agents.

Discharging sinus and the underlying bone: micro-organisms involved A clinician treating a patient with a suspected infected wound will usually send a superficial pus swab to the laboratory and institute antibiotic therapy accordingly. Particular problems, however, exist for wounds that are in fact sinuses overlying a focus of chronic osteomyelitis. Mackowiack in 1978 demonstrated the low positive predictive value of sinus tract swabs; in a retrospective analysis of 40 patients, only $44 \%$ of the soft tissue swabs contained the same pathogen as that obtained from a bone sample at the time of operation. However, isolation of Staphylococcus aureus from the sinus tract swab had a higher positive predictive value than for other bacteria $(78 \%)$. The same study found $60 \%$ of chronic osteomyelitis to be due to $S$ aureus, followed by enterobacteriaceae $(23 \%)$, pseudomonas $(9 \%)$ and streptococcus $(9 \%){ }^{3}$ Similar results with regards to the inaccuracy of wound swabs were obtained in prospective studies on diabetic foot infection ${ }^{4}$ and on post-traumatic chronic osteomyelitis. ${ }^{5}$ The latter study also demonstrated the poor positive predictive value of Craig needle biopsy specimens. Accurate identification of the micro-organism and its sensitivity can therefore only be obtained by an open surgical procedure, and there appears little (if any at all) place for non-invasive diagnostic methods.

From cultures of operative specimens, $S$ aureus is the main causative agent of chronic bacterial osteomyelitis, accounting for about two thirds of the isolates, followed by pseudomonas and enterobacteriaceae. ${ }^{356}$ Osteomyelitis complicating diabetic foot infection, however, is typically polymicrobial, with mixed aerobes, facultative anaerobes, and strict anaerobes being isolated at operation. ${ }^{4}$ Coagulase negative Staphylococcus spp, such as $S$ epidermidis, are responsible for the majority of chronic osteomyelitis associated with orthopaedic implants and for $90 \%$ of pin tract infections. ${ }^{78}$ Other less common micro-organisms isolated include salmonella (more frequent in developing countries), and anaerobes such as clostridium and Pasteurella multocida. ${ }^{4} 69$

Box 1 summarises the above section.

\section{Box 1: Micro-organisms}

- The typical causative agent is $S$ aureus, or $S$ epidermidis if associated with an implant. Gram negative bacteria are present in about one third of cases.

- Chronic osteomyelitis in the diabetic foot is polymicrobial with mixed Gram positive and Gram negative bacteria (aerobes and anaerobes).

- Wound swabs are all too often inaccurate in chronic osteomyelitis: cultures from operative specimens are a more reliable method of planning antibiotic treatment. 
Pathophysiology of bone infection

Rodet produced the first experimental acute haematogenous osteomyelitis in 1884: injection of rabbits with "un micrococcus qu'il possede une couleur jaune orange" ( $S$ aureus) resulted in the occurrence of multiple bone abscesses. ${ }^{10}$ Since then various animal models have been studied, including rodents, chicks, rabbits, dogs ${ }^{11}$ and, more recently, sheep. ${ }^{12}$ The development and progression of the disease can be summarised in bacterial contamination and adhesion followed by infection and subsequent chronicity. Patient predisposing factors intervene at various levels (fig 1).

PATIENT PREDISPOSING FACTORS

Healthy bone tissue is extremely resistant to infection. ${ }^{13}{ }^{14}$ The presence of bone necrosis, heavy contamination or foreign bodies, as well as general predisposing factors such as diabetes and peripheral vascular disease tip the balance in favour of the bacterium.

Trauma or surgery can produce devitalised bone fragments. The other single most potent bone necrotising factor is indeed ischaemia. In the chick model of haematogenous osteomyelitis, patchy ischaemic bone necrosis occurs when the infective process occludes the vascular tunnels. This creates an ideal culture medium for bacteria, and at 48 hours, abscesses are formed. A sequestrum develops within eight days. ${ }^{15}$

The role of bone necrosis is pivotal to the establishment of experimental chronic osteomyelitis by direct inoculum: Norden and Kennedy in 1970 used intramedullary sodium morrhuate, a sclerosing agent, before direct inoculation of $S$ aureus in order to obtain osteomyelitis in rabbits. Inoculation of bacteria without sodium morrhuate or vice versa failed to produce an infection. ${ }^{16}$

BACTERIAL CONTAMINATION AND ADHESION.

DEVELOPMENT OF INFECTION

Bacteria may reach the bone via the bloodstream, by direct inoculum caused by, for

Bacterial contamination (direct inoculus, intraoperative contamination, haematogenous)

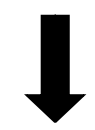

Bacterial adhesion to bone or implant

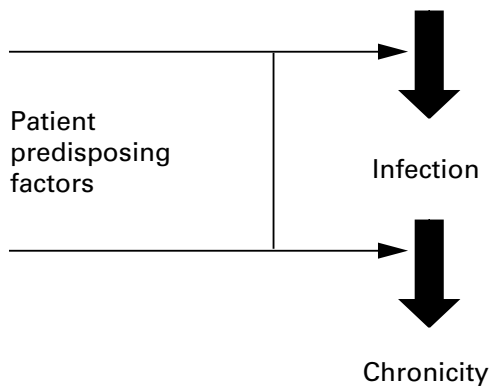

Figure 1 Pathogenesis of chronic bacterial osteomyelitis.

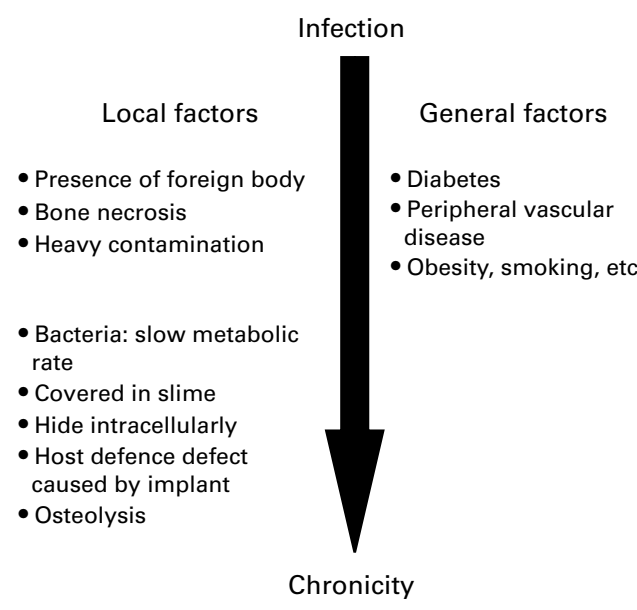

Figure 2 Pathogenetic factors behind the chronicity of a bone infection.

example, trauma or surgery, or by direct spread from an adjacent soft tissue infection (for example diabetic foot ulcer or periodontal disease). Whatever the route of access, bacteria must be able to subsequently adhere to components of the bone matrix in order to start the infection.

$S$ aureus has been known to bind fibrinogen for some decades. This may well provide an explanation for the ability of this microorganism to survive in body fluids. Bacteria clump together and are covered in a layer of fibrinogen, thus protected from host defence mechanisms and antibiotics. ${ }^{17}$ However, this alone does not explain how bacteria adhere to bone matrix. Staphylococcus spp express high affinity receptors (adhesins) for fibronectin, ${ }^{18} 19$ collagen, ${ }^{20}$ and laminin. ${ }^{21}$

Fibronectin, a glycoprotein found in many body fluids and connective tissue matrices, appears to be particularly relevant to the pathogenesis of chronic osteomyelitis: bacterial adherence to polymers similar to the ones used in orthopaedic surgery is mediated by fibronectin. ${ }^{22}{ }^{23}$ Fibronectin, fibrinogen, and laminin have been demonstrated to be responsible for adherence of $S$ aureus to the surface of a foreign body in an animal model. ${ }^{24}$ The same glycoprotein has been shown to mediate bacterial adhesion to metal plates and screws. ${ }^{25}$

Sublethal doses of antibiotics have been shown to inhibit mucosal adhesion by group A streptococci and Escherichia coli. ${ }^{26}$ This is an interesting finding that may provide part of the biological explanation for the efficacy of prophylactic intravenous antibiotics in orthopaedic surgery.

CHRONICITY ASSOCIATED WITH AN IMPLANT

Persistence of bone infection will result in chronic osteomyelitis. The main factors responsible for the development of chronicity are listed in fig 2.

Bacterial persistence is the rule in chronic osteomyelitis associated with a foreign body such as plate and screws or joint replacement. In such cases antimicrobial therapy alone is often unsuccessful, and the infection is cured only by implant removal and debridement of necrotic bone. ${ }^{27}$ 
Southwood et al, working with an animal model (rabbit) of orthopaedic implant infection, found that as few as 50 organisms contaminating the operative site after a cemented hemiarthroplasty had been implanted resulted in infection, whereas 10000 organisms were necessary to produce infection in the absence of a foreign body. ${ }^{28}$ After adherence on the implant via fibronectin receptors, usually on surface irregularities, coagulase negative staphylococci develop a slimy coat that protects the colony from host defence mechanisms and antibiotics. ${ }^{29}$ There is also evidence for a local host defence defect in the pathogenesis of foreign body infection. Polymorphonuclear leucocytes (PMLs) extracted from tissue fluid surrounding a foreign body are unable to kill catalase positive $S$ aureus despite optimal opsonisation. ${ }^{30}$ The same PMLs also exhibit a decreased production of superoxide and have lower content of enzymatic granules, an indication of impaired response to infection. The in vitro interaction of PMLs with Teflon leads to respiratory burst and exocytosis of enzymatic granules. The end result is a PML population composed of exhausted cells, with lower granule content, and less killing capacity. ${ }^{30}$ A similar study also demonstrated that also the opsonisation of $S$ aureus in the presence of a foreign body is dramatically reduced at 20 hours. ${ }^{31}$ Since $S$ aureus is mainly killed by opsonisation and subsequent phagocytosis by PMLs, the host defence defects described above are particularly relevant to the development of chronic osteomyelitis associated with internal fixation or joint replacement.

Box 2 summarises the above section.

CHRONICITY NOT ASSOCIATED WITH AN IMPLANT Chronic osteomyelitis has been shown to result from persistence of the acute haematogenous form in $4.4 \%$ of children in a recent Scottish study. ${ }^{32}$ Waldvogel et al in 1970 reported development of chronicity in $15 \%$ of adults. ${ }^{2}$

Chronic osteomyelitis may present as a recurrent or intermittent disease, with periods of quiescence of variable duration. Relapses of the disease several decades after the acute episode are well known (fig 3). ${ }^{33}$ Late reactivation

\section{Box 2: Persistence of infection 1}

- Healthy bone is extremely resistant to infection.

- However, once an infection has established, its eradication by antibiotic treatment is very difficult.

- Dead bone and implants are the most common reasons for failure of conservative treatment. Under those circumstances bacteria cannot be reached by host defence mechanisms or antibiotics.

- The presence of an implant causes exhaustion of local polymorphonuclear cells that become unable to kill phagocytosed bacteria.

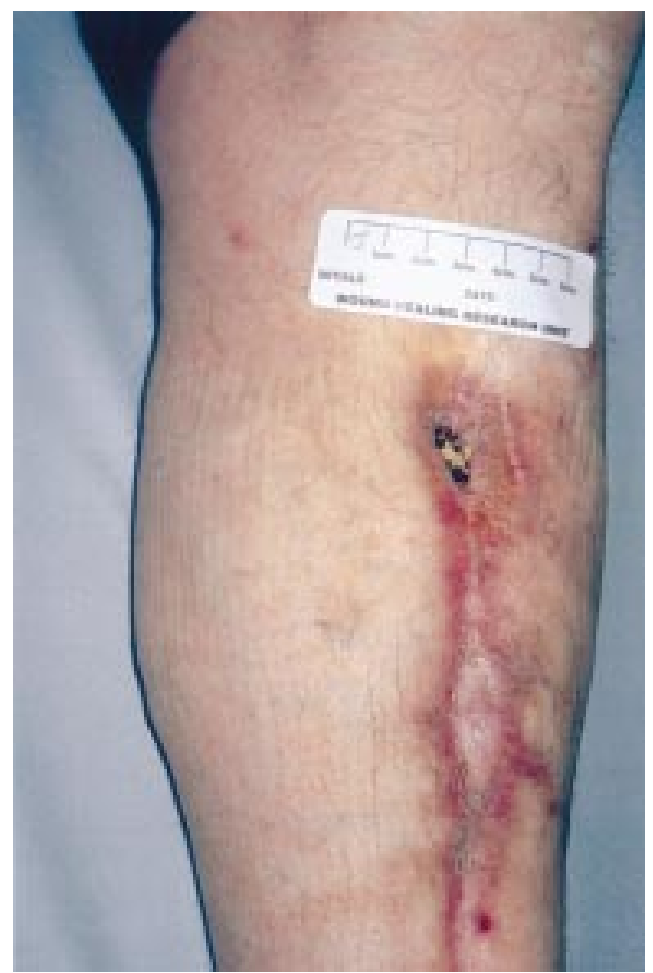

Figure 3 Lower leg of patient with non-healing wound (see case report). The linear scar on the leg is the result of an open drainage procedure carried out when he was a child.

of osteomyelitis up to 80 years after the primary illness had been "cured" have been reported. ${ }^{34}{ }^{35}$ In a series of 12 patients with a minimum latent period of 20 years, no definite predisposing factor was identified, and both haematogenous and post-traumatic infections seemed equally to be involved. ${ }^{36}$

It is easy to understand the reasons behind the failure of medical treatment of osteomyelitis in the presence of dead bone: the infection will self perpetuate until all sequestra have been debrided. But what causes the same failure of antibiotic treatment in healthy individuals, with normal host defence mechanisms and negative radiology for abscesses or sequestra? And what causes late reactivation? Is osteomyelitis a lifelong disease?

$S$ aureus, mainly non-encapsulated variants, can be internalised by chick osteoblasts ${ }^{37}$ and endothelial cells ${ }^{38}$ in vitro and survive intracellularly, protected from host defence mechanisms and antibiotics. This might explain the known problem of a flare up of osteomyelitis with no identifiable causative organism. Furthermore, staphylococci can also acquire a very slow metabolic rate, in a phenotypic alteration named small colony variant. Slow growing bacteria have been known to be resistant to antibiotics since 1942, active cell wall synthesis being necessary for penicillin to be bactericidal. $^{39} 40$ Small colony variants of $S$ aureus were described for the first time in 1932 by Hoffstadt and Youmans as minuscule bacterial colonies (less than $1 \mathrm{~mm}$ ) that grew very slowly and often required magnification to be seen. ${ }^{41}$ Small colony variants were found to be resistant to penicillin one year after its 
discovery by Fleming. ${ }^{39}$ Small colony variants may indeed account for the frequent failure to identify the causative micro-organism in chronic osteomyelitis: these strains may be easily missed or overgrown in a busy laboratory. They may also account for the frequent clinical presentation of chronic osteomyelitis as a slow, indolent infection that causes little inflammatory response and persists despite prolonged antimicrobial therapy.

Box 3 summarises the above section.

\section{BACTERIALLY INDUCED OSTEOLYSIS}

During the course of infection, bacteria induce local bone destruction (osteolysis). This aids the spread and persistence of infection and is responsible for the septic loosening of an implant. It is not to be confused with bone loss secondary to ischaemia. An acute infection will cause an intense inflammatory response, thrombosis of endosteal and periosteal vessels, bone infarcts with subsequent abscess and sequestrum formation. A slow, indolent infection will produce a mild to moderate inflammatory response and little or no ischaemic necrosis. A balance will be achieved between bone resorption and new bone formation, and sequestra are less likely to develop.

Osteolysis is accomplished by osteoclasts via stimulation by soluble factors (fig 4). These in turn are produced by immune system cells after interaction with bacteria. Factors such as Gram negative endotoxin, its lipopolysaccharide fraction and $\mathrm{N}$-acetyl-muramyl-dipeptide (MDP) are potent osteolytic factors in vitro. ${ }^{42-44}$ Furthermore, endotoxin and MDP have been shown to stimulate bone resorption in cultured long bones. ${ }^{45}$

Staphylococcal surface proteins have a potent osteolytic effect. Surface proteins from $S$ aureus can be inhibited by indomethacin, by antibodies to interleukin-1 (IL-1) receptors or tumour necrosis factor (TNF). Surface proteins from $S$ epidermidis act by a prostaglandinindependent mechanism, are blocked by a neutralising antibody to $\mathrm{TNF}-\alpha$ and only partially by anti-IL-1..$^{45-49}$ Also streptococcal cell wall components can stimulate bone resorption and inhibit protein synthesis in cultured mouse calvariae by a prostaglandin independent mechanism. ${ }^{49}$

\section{Box 3: Persistence of infection 2}

- Chronic osteomyelitis is often a lifelong disease. Late reactivation up to 80 years after the primary episode has been reported.

- Bacteria can also elude host defence mechanisms by hiding intracellularly and by developing a protective slimy coat.

- By acquiring a very slow metabolic rate, bacteria become less sensitive to antibiotics.

- For all the above reasons, operative treatment should be considered whenever possible.

\section{Case report}

A 60 year old man presented with a non-healing wound on his lower leg of several weeks' duration (fig 3).

Inflammatory markers were raised and imaging studies were consistent with chronic osteomyelitis.

When he was a child he had successfully undergone treatment for acute osteomyelitis at the same site. The linear scar on the leg is in fact the result of a drainage procedure. The disease had remained quiescent for over 50 years!

The so-called osteoclast activating factor is in fact a mixture of cytokines, of which the most potent is IL-1; TNF and lymphotoxin are less powerful but have a synergistic interaction with IL-1..$^{50}$ 5-Lypoxygenase metabolites of arachidonic acid also stimulate osteoclasts, and this might offer an opportunity for pharmacological therapy in the future. ${ }^{4251}$

Leucocyte derived factors such as TNF induce osteoblasts to cooperate in bone resorption by stimulating osteoclastic activity via a soluble factor. ${ }^{52}$ This factor is probably IL-6..$^{53}$

Another recent and fascinating finding is that extracts of $S$ aureus and $S$ epidermidis cause decreased bone matrix formation in vitro, thereby suggesting that impaired osteogenesis might be an important element of staphylococcal osteomyelitis. ${ }^{54}$

\section{Conclusion}

Chronic bacterial osteomyelitis remains a major challenge despite advances in antibiotic

Bacterial surface antigens (LPS, staphylococcal surface associated proteins, etc)

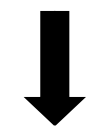

Lymphocytes, macrophages

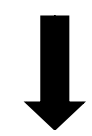

Cytokines (IL-1, IL-6, TNF- $\alpha$ and TNF- $\beta$ )

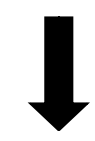

Increased osteoclastic and decreased osteoblastic activity

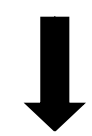

Bone tissue loss

Figure 4 Process of osteolysis. Osteolysis is mediated by soluble factors produced by immune cells after interaction with bacterial antigens. This in turn both increases osteoclastic and decreases osteoblastic activity (LPS = lipopolysaccharide). 
engineering and better aseptic techniques in the operating theatre. Bacteria (predominantly $S$ aureus and $S$ epidermidis) adhere to bone matrix and orthopaedic implants via receptors to fibronectin and to other structural proteins. They subsequently elude host defences and antibiotics by "hiding" intracellularly, by developing a slimy coat or by acquiring a very slow metabolic rate. The presence of an orthopaedic implant also causes a local polymorphonuclear cell defect, with decreased ability to kill phagocytosed bacteria.

Osteolysis is determined locally by the interaction of bacterial surface components with immune system cells and subsequent cytokine production. Numerous soluble mediators are involved, the end result being both increased osteoclastic activity and decreased new bone formation by osteoblasts.

The increasing development of antibiotic resistance by $S$ aureus and $S$ epidermidis will probably make conservative treatment even less successful than it is now. A close interaction between orthopaedic surgeons and physicians, with combined medical and operative treatment, is to be commended.

1 Joyner AL, Smith DT. Acute staphylococcus osteomyelitis. Surg Gynecol Obstet 1936;63:1-6.

2 Waldvogel FA, Medoff G, Swartz MN. Osteomyelitis: a review of clinical features, therapeutic considerations and
unusual aspects (first of three parts). $N$ Engl $\mathcal{F}$ Med unusual aspects (first

3 Mackowiack PA, Jones SR, Smith JW. Diagnostic value of inus-tract cultures in chronic osteomyelitis. $F A M A 1978$ 239:2772-5

4 Wheat LJ, Allen SD, Henry M, et al. Diabetic foot infections - bacteriologic analysis. Arch Intern Med 1986; 146:1935-40.

5 Perry CR, Pearson RL, Miller GA. Accuracy of cultures of material from swabbing of the superficial aspect of the wound and needle biopsy in the properative assessment of osteomyelitis. F Bone foint Surg Am 1991;73:745-9.

6 Haas DW, McAndrew MP. Bacterial osteomyelitis in adults: evolving considerations in diagnosis and treatment. $A m \mathcal{F}$ Med 1996;101:550-61

7 Quie PG, Belani KK. Coagulase negative staphylococcal adherence and persistence. F Infect Dis 1987;156:543-7.

8 Mahan J, Seligson D, Henry SL, et al. Factors in pin-tract Mahan J, Seligson D, Henry SL, et al.
infections. Orthopedics 1991;14:305-8.

9 Baker GL, Oddis CV, Medsger Jr TA. Pasteurella multocBaker GL, Oddis CV, Medsger Jr TA. Pasteurella multoc-
ida. Polyarticular septic arthritis. F Rheumatol 1987;14:355-

10 Rodet A. Etude experimentale sur l'osteomyelite infectieuse. Comptes Rendus de l'Academie des Sciences 1884;99: 569-71.

11 Norden CW. Lessons learned from animal models of osteomyelitis. Rev Inf Dis 1988;10:103-10.

2 Kaarsemaker S, Walenkamp GH, v d Bogaard AE. New model for chronic osteomyelitis with Staphylococcus aureus in sheep. Clin Orthop 1997;339:246-52.

13 Scheman L, Janota M, Lewin P. The production of experimental osteomyelitis. $7 A M A$ 1941;117:1525-9.

14 Andriole VT, Nagel DA, Southwick WO. A paradigm for human chronic osteomyelitis. F Bone f Surg Am 1973; 55:1511-15.

15 Emslie KR, Nade S. Acute haematogenous staphylococcal osteomyelitis: a description of the natural history in an avian osteomyelitis: a description of the natu
model. Am f Physiol 1983;110:333-45.

16 Norden CW, Kennedy E. Experimental osteomyelitis. A description of the model. F Infect Dis 1970;122:410-18.

17 Duthie ES. The action of fibrinogen on certain pathogenic cocci. F Gen Microbiol 1955;13:383-93.

18 Kuusela P. Fibronectin binds to Staphylococcus aureus. Nature 1978;276:718-20.

19 Estersen F, Clemmensen I. Isolation of a fibronectinbinding protein from Staphylococcus aureus. Infect Immun 1982;37:526-31.

20 Patti JM, Boles JO, Hõõk M. Identification and biochemical characterization of the ligand binding domain of the collagen adhesin from Staphylococcus aureus. Biochemistry 1993;32:11428-35.

21 Lopes JD, Dos Reis M, Brentani RR. Presence of laminin receptors in Staphylococcus aureus. Science 1985;299:275-7.

22 Maxe I, Ryden C, Wadstrõm T, et al. Specific attachment of Staphylococcus aureus to immobilized fibronectin. Infect Immun 1986;54:695-704.

23 Vaudaux PE, Waldvogel FA, Morgenthaler JJ, et al. Adsorption of fibronectin onto polymethylmethacrylate and
promotion of Staphylococcus aureus adherence. Infect promotion of Staphylo
24 Herrmann M, Vaudaux PE, Pittet D, et al. Fibronectin, fibrinogen and laminin act as mediators of adherence of clinical staphylococcal isolates to foreign material. F Infect Clinical staphylococcal

25 Fischer B, Vadaux P, Magnin M, et al. Novel animal model for studying the molecular mechanisms of bacterial adhesion to bone-implanted metallic devices: role of fibronectin in Staphylococcus aureus adhesion. F Orthop Res 1996;14:914-20.

26 Alkan ML, Beachey EH. Excretion of lipoteichoic acid by group A streptococci: influence of penicillin on excretion and loss of ability to adhere to human oral mucosal cells. $\mathcal{f}$ Clin Invest 1978;61:671-7.

27 Anonymous. Infected hip prosthesis (editorial). BM7 1980; 280:1241-2.

28 Southwood RT, Rice JL, Mc Donald PJ, et al. Infection in experimental hip arthroplasties. F Bone foint Surg Br 1985;67:229-31

29 Peters G, Locci R, Pulvere G. Adherence and growth of coagulase-negative staphylococci on surfaces of intravenous catheters. F Infect Dis 1982;146:479-82.

30 Zimmerli W, Lew PD, Waldvogel FA. Pathogenesis of foreign body infection-evidence for a local granulocyte defect. $\mathcal{F}$ Clin Invest 1984;73:1191-200.

31 Zimmerli W, Waldvogel FA, Vaudaux P, et al. Pathogenesis of foreign body infection: description and characteristics of an animal model. F Infect Dis 1982;146:487-97.

32 Craigen MAC, Wattera J, Hackett JS. The changing epidemiology of osteomyelitis in children. $\mathcal{F}$ Bone foint Surg $\mathrm{Br}$ 1992;74:541-5.

33 Scully RE, Mark EJ, McNeely WF, et al. Case records of the Massachusetts General Hospital-case 6. N Engl f Med 1993;328:422-8

34 Korovessis P, Fortis AP, Spastris P, et al. Acute osteomyelitis of the patella 50 years after a knee fusion for septic arthritis: a case report. Clin Orthop 1991;272:205-7.

35 Gallie WE: First recurrence of osteomyelitis eighty years after infection. F Bone foint Surg Br 1951;33:110-1.

36 Perko M, Taylor TKF, Ruff SJ. Late reactivation of osteomyelitis in long bones. Proceedings of the 43rd Australian Orthopaedic Association. 7 Bone foint Surg Br 1984; 66:782.

37 Hudson MC, Ramp WK, Nicholson NC, et al. Internalization of Staphylococcus aureus by cultured osteoblasts. Microb Pathog 1995;19:409-19.

38 Balwit JM, v Langevelde P, Vann JM, et al. Gentamycin resistant menadione and hemin auxotrophic Staphylococcus aureus persist within cultured endothelial cells. F Infect Dis 1994;170:1033-7.

39 Schnitzer RJ, Camagni LJ, Buck M. Resistance of small colony variants ( $\mathrm{G}$-forms) of a Staphylococcus towards the bacteriostatic activity of penicillin. Proc Soc Exp Biol Med 1943;53:75-8.

40 Czens RM, Tuomanen E, Tosch W, et al. Evaluation of the bactericidal activity of $\beta$-lactam antibiotics on slowly growing bacteria cultured in the chemostat. Antimicrob Agents Chemother 1986;29:797-802.

41 Hoffstadt RE, Youmans GP. Staphylococcus aureus dissociation and its relation to infection and immunity. $\mathcal{F}$ Infect Dis 1932;51:216.

42 Nair SP, Meghji S, Wilson M, et al. Bacterially induced bone destruction: mechanisms and misconceptions. Infect Immun 1996;64:2371-80.

43 Hausmann E, Raisz L, Miller WA. Endotoxin: stimulation of bone resorption in tissue culture. Science 1970;168:862-4.

44 Dewhirst F. N-Acetyl muramyl dipeptide stimulation of bone resorption in tissue culture. Infect Immun 1982;35: $133-7$.

45 Raisz LG, Alander C, Eilon G, et al. Effects of two bacterial products, muramyl dipeptide and endotoxin, on bone resorption in org an cultur

46 Nair S, Song Y, Meghji S, et al. Surface-associated proteins from Staphylococcus aureus demonstrate potent bone resorbing activity. $\mathcal{F}$ Bone Miner Res 1995;10:726-34

47 Nair SP, Megji S, Wilson M, et al. Clinical isolates of Staphylococcus aureus have osteolytic surface proteins and a proportion of the population have antibodies that block this activity: is this of prognostic significance? Br F Rheumatol 1997;36:328-32.

48 Megji S, Crean SJ, Nair S, et al. Staphylococcus epidermidis produces a cell-associated proteinaceous fraction which causes bone resorption by a prostanoid-independent mechanism: relevance to the treatment of infected orthopaedic implants. Br $\mathcal{F}$ Rheumatol 1997;36:957-63.

49 Meikle MC, Gowen M, Reynolds JR. Effects of streptococcal cell wall components on bone metabolism in vitro. Calcif Tissue Int 1982;34:350-64.

50 Stashenko P, Dewhirst FE, Peros WJ, et al. Synergistic interactions between interleukin-1, tumor necrosis factor and lymphotoxin in bone resorption. f Immunol 1987;138:1464-

51 Gallwitz WE, Mundy GR, Lee CH, et al. 5-Lipoxygenase metabolites of arachidonic acid stimulate osteoclasts to resorb calcified matrices. F Biol Chem 1993;268:10087-94. 2 Thomson BM, Mundy GR, Chambers TJ. Tumor necrosis factors alpha and beta induce osteoblastic cells to stimulate

53 Ishimi Y, Miyaura C, He Jin C, et al. IL-6 is produced by osteoblasts and induces bone resorption. $\mathcal{f}$ Immunol 1990;145:3297-303.

54 Lerner UH, Sundqvist G, Ohlin A, et al. Bacteria inhibit biosynthesis of bone matrix proteins in human osteoblasts. Clin Orthop 1998;346:244-54. 